\title{
Feasibility of Vis/NIR spectroscopy and image analysis as basis of the development of smart-drying technologies
}

\author{
Sturm, B. ${ }^{\text {a*}}$; Moscetti, R. ${ }^{\text {b; Crichton, S.O.J. }}$; Raut, S. ${ }^{\text {a }}$ Bantle, M, c; Massantini, R. ${ }^{\text {b }}$ \\ ${ }^{a}$ Process and Systems Engineering in Agriculture Group, Department of Agricultural and Biosystems \\ Engineering University of Kassel, Witzenhausen, Germany \\ ${ }^{\mathrm{b}}$ Department for Innovation in Biological, Agro-food and Forest system (DIBAF), \\ Tuscia University, Viterbo, Italy \\ ${ }^{c}$ Department of Energy Processes, SINTEF Energy Research, Trondheim, Norway \\ *E-mail of the corresponding author: barbarasturm@daad-alumni.de
}

\begin{abstract}
Drying is a complex, dynamic, unsteady and nonlinear process that, when not optimized on a system level, may be responsible for (1) significant quality degradation and (2) energy wastage. Consequently, new drying technologies must be designed combining non-invasive at-/on-/in-line advanced measurement and control systems with models cross-linking all relevant aspects of product quality changes and heat and mass transfer phenomena.

This paper presents preliminary results on the use of RGB imaging, NIR spectroscopy and Vis-NIR hyperspectral imaging for real-time monitoring of physicochemical changes of apples and carrots during drying.
\end{abstract}

Keywords: chemometrics, artificial intelligence, deep learning 


\section{Introduction}

Commercial drying of apple and carrot produce is generally conducted according to drying strategies developed decades ago. These strategies operate to a large degree based on strict control of the characteristics of raw material at the input stage. This strict sorting, however, results in a significant proportion of produce being wasted prior to any processing. This coarse sorting at the input stage, however, does not guarantee that, after the set drying process, the entire produce will be of the same quality or will even fulfill the minimum quality required by consumers. In recent years, the organic sector has put significant efforts into the development of clear definitions for gentle and quality oriented ways of processing plant based foodstuffs to supplement existing regulations and guarantee premium quality of the product whilst reducing resource depletion [1].

The use of non-invasive measurement and control systems have shown a great potential for improvement of the product quality [2]. Simple solutions can readily be implemented into existing processes (e.g. dynamic control of product temperature [3]), while integration of advanced solutions is not possible nor financially viable in practice. Thus, there is a need for smart processing systems which allow for simultaneous multi-factor control (i.e. air temperature, velocity, humidity) to guarantee high-value end products, while enhancing energy demand and resource efficiency, by implementing innovative and reliable microcontrollers, sensors, resources, tools and practices at low cost and embracing various R\&D areas (e.g. computer vision, hyper-/multispectral imaging, deep learning, etc.) [4].

Aim of this study was, therefore, the development of non-invasive measurement systems based on RGB imaging, Vis/NIR Hyperspectral imaging and NIR spectroscopy in combination with analysis algorithms to determine colour development, water content and chemical components throughout the drying process. These technologies and techniques are essential to provide inputs for the development of product quality driven adaptive control systems for drying. Eventually, these systems are intended to be transferred to simpler solutions and integrated into smart control systems for product quality oriented drying.

\section{Materials and Methods}

\subsection{RGB imaging study on apples}

Organic apples var. Red Delicious with the origine Italy were sourced from a local supplier (Trondheim, Norway) and stored at $8^{\circ} \mathrm{C}$ until processing. For the trials slices of $5 \mathrm{~mm}$ were cut. Core and skin were not removed.

Drying experiments were conducted at 20 and $40{ }^{\circ} \mathrm{C}$ with relative air humidities of 25,40 and $60 \%$ using an experimental drying chamber built at SINTEF. The chamber was equipped with a camera system, including an LED barlight for illumination. Details on the experimental set-up and conduction of trials are presented by Bantle [5]. 
Analysis of optical alterations was conducted using the OpenCV libraries for image processing, using images taken every 5 minutes during the drying tests. RGB values were transformed into CIE-XYZ color space according to the ISO Standrad 13655 and then further processed to receive the CIE-L*a*b values. A detailed procedure is given by Bantle et al. [5].

\subsection{Vis/NIR Hyperspectral imaging study on apples}

Apples var. Golden Delicious were sourced from a local retailer (Witzenhausen) and stored at room temperature until use. The apples were de-cored and sliced to $5 \mathrm{~mm}$ thickness and cut to a uniform outer diameter of $65 \mathrm{~mm}$. Drying was conducted in a hot air convection oven at $50^{\circ} \mathrm{C}$.

Drying tests were conducted using a convective air cabinet dryer (HT-Mini, Innotech Ingenieurgesellschaft mbH, Altdorf, Germany) at 50 and $70^{\circ} \mathrm{C}$. Measurement protocols followed those described by Crichton et al. [6].

Samples were imaged using a Specim V10E PFD hyperspectral camera (Schneider Optics Xenoplan 1.9/35) in conjunction with a linear translation stage (Specim Spectral Imaging Ltd., Finland). Three 60W halogen GU10 bulbs were used for illumination. A detailed description of the image processing moisture content prediction protocols followed is given by Crichton et al. [6].

\subsection{Near infrared spectroscopy study on carrots and apples}

Organic carrots (Daucus carota L., var. Romance) and apples (Malus domestica B. var. Gala) were purchased and immediately stored at $4 \pm 1^{\circ} \mathrm{C}$ until processing. Carrot slices and apple wedges were prepared by washing and cutting samples using a sharp ceramic knife. The slices and wedges were subjected to 8-h hot-air drying and batch sampling was performed at every hour of drying. The dehydration process was conducted at $40^{\circ} \mathrm{C}$ (carrot) and $60^{\circ} \mathrm{C}$ (apple). Samples were divided into two groups, one of which was subjected to a blanching pre-treatment before hot-air drying and one that was dried without pre-treatment (i.e. hot-water blanching at $95^{\circ} \mathrm{C}$ for $1.45 \mathrm{~min}$ for carrots and microwave blanching at 850W for 45 sec for apples). Each batch was subjected to both NIR spectral data acquisition and determination of CIELab colour, moisture content (wet basis), water activity $\left(a_{w}\right)$, soluble solids content and total carotenoid content (carrot only).

Absorbance spectra were acquired using a spectrophotometer mod. Luminar 5030 (Brimrose Corp., Baltimore, USA) (range 1100-2300 nm, 2-nm resolution). Each sample was measured in duplicate on two opposite sides of each slice/wedge (i.e. 4 spectra per sample).

Chemometric analysis was performed following spectral pre-treatments including Standard Normal Variate, Multiplicative Scatter Correction, Savitzky-Golay first and second 
derivatives with a second or third order polynomial fitted over a window of five, seven, nine or eleven features, as well as Mean Centering [7, 8].

Regression models were computed using the partial least squares (PLS) regression through the SIMPLS algorithm [9]. The samples were randomly split as calibration set (C) and the prediction set $(\mathrm{P})$. The venetian blinds cross-validation with 10 data splits was performed. Root Mean Square Error (RMSE), BIAS and coefficient of determination $\left(R^{2}\right)$ were employed as model performance metrics [10]. PLS models were computed to predict changes in chemical and physicochemical attributes during 8-h dehydration process.

\section{Results}

\subsection{RGB imaging-based analysis of color changes in apples}

The employed camera system and associated developed codes allowed for the reliable realtime measurement of changes in product quality at the set measurement intervals. Fig. 1 shows the impact of drying conditions on the resulting changes in the $\mathrm{a}^{*}$ and $\mathrm{b}^{*}$ values. It is evident that all settings led to an increase of both $a^{*}$ and $b^{*}$ values, signifying the change of visual appearance of the samples. The most beneficial drying conditions for maintenance of the appearance were at $20^{\circ} \mathrm{C}(\mathrm{T})$ and $25 \% \mathrm{RH}$.

The developed real-time monitoring system for color (and shape) changes will make it possible to include information of the current color changes in the control consideration of a smart drying system. This is of particular importance as consumers tend to judge a product's quality by its visual appearance.

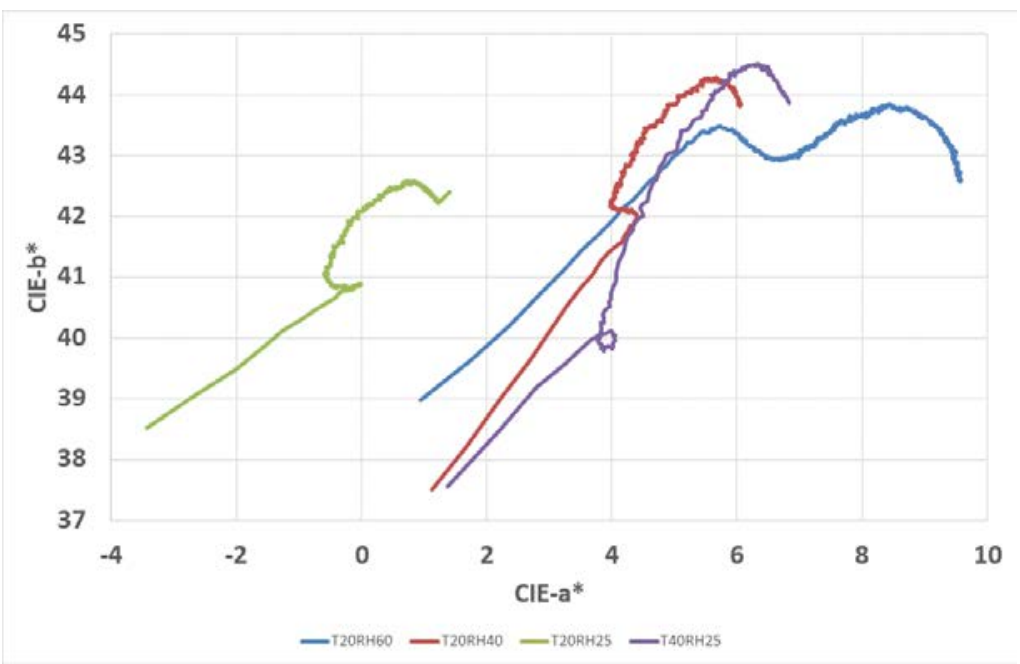

Fig 1: Development of CIE-a* as a function of CIE-b* for different drying conditions, tempertures are given as $T$ and relative humidity as $R H$ in the graph 


\section{Vis/NIR hyperspectral water content determination in apples}

PLSR analyses with the full reflectance spectra were carried out across the complete data set. For both conditions PLSR was performed for models varying from 1-20 components with 10-fold creation and testing. The 3-component [540 nm, $817 \mathrm{~nm}, 977 \mathrm{~nm}$ ] PLSR model satisfied the minimal RMSE criterion. Table 1 gives an overview of the results for full and reduced spectra.

Table 1: Performance of moisture content PLSR models

\begin{tabular}{|c|c|c|c|c|c|c|c|c|}
\hline \multicolumn{5}{|c|}{ Full Spectrum } & \multicolumn{4}{|c|}{ Reduced (3-wavelength set) } \\
\hline Setting $\left({ }^{\circ} \mathrm{C}\right)$ & Calibı & ration & Test & & Calib & oration & Test & \\
\hline & $r^{2}$ & $\begin{array}{l}\text { RMSE (g/g) } \\
\text { [M.C. \%] }\end{array}$ & $r^{2}$ & $\begin{array}{l}\text { RMSE (g/g) } \\
\text { [M.C. \%] }\end{array}$ & $r^{2}$ & $\begin{array}{l}\text { RMSE (g/g) } \\
\text { [M.C. \%] }\end{array}$ & $r^{2}$ & $\begin{array}{l}\text { RMSE (g/g) } \\
\text { [M.C. \%] }\end{array}$ \\
\hline 50 & 1.00 & $0.01[0.12]$ & 1.00 & $0.11[1.32]$ & 0.99 & $0.18[2.16]$ & 1.00 & $0.09[1.08]$ \\
\hline 70 & 1.00 & $0.03[0.36]$ & 1.00 & $0.07[0.84]$ & 1.00 & $0.18[2.16]$ & 0.98 & $0.05[0.60]$ \\
\hline
\end{tabular}

For the deeper understanding of the drying behavior of foodstuffs in terms of heat and mass transfer, and the performance analysis of the applied drying devices, a spatial and temporal evaluation of moisture content development during the drying process is intrinsic. Thus, the spatial variation of reflectance spectra at different times of the drying process was used to visualize the development of moisture content throughout the drying process as well as the spatial difference of said moisture content (Fig. 2).
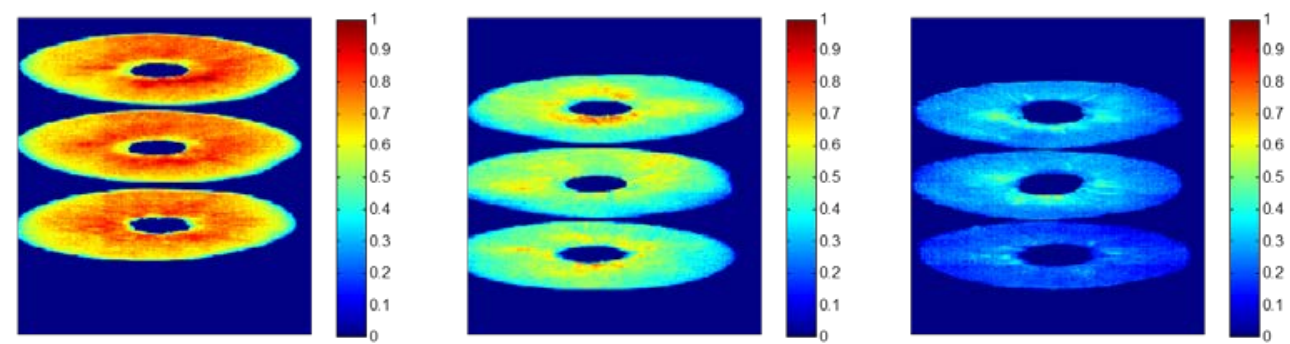

Fig 2: Normalised moisture distribution for apple slices before (left), during (middle) and at the end of the drying process.

Further studies using hyperspectral imaging on apples [11], meat [12] and potatoes [13, 14] show similarly promising results. A reduction of the wavelengths needed for detection of product features will allow for the transfer to simpler systems, e.g. colour or black and white cameras in combination with either selective lighting or band pass filters. This could then also be combined with full color detection described above, as well as physical feature detection, as described by Bantle et al. [5] if sequential feature detection is implemented. This method will in turn open new possibilities for deeper analyisis of the drying process and consequentlythe integration of feature analysis into smart drying process control. 


\subsection{Near infrared spectroscopy study on carrots and apples}

\subsubsection{Regression models (carrots)}

Poor results $\left(R^{2}<0.70\right)$ were obtained for the prediction of soluble solids content for the unblanched treatment. Conversely, regression models with good $\left(R^{2} \geq 0.80\right)$, very good $\left(R^{2} \geq\right.$ $0.90)$ or excellent $\left(R^{2} \geq 0.95\right)$ predictability were obtained to monitor changes in soluble solids content (only for blanched carrots), $a_{w}$ (Fig. 3a), moisture content (Fig. 3b), total carotenoids content (Fig. 3c) and color changes (Fig. 3d) of carrot slices during drying, regardless of thermal treatment. Except for the calibration model for color changes, the coefficient of determination $\left(R^{2}\right)$ was higher than 0.90 for the parameters. Thus, for the test set validation with one quarter of the data (randomly selected) RMSEPs ranging from 0.03-0.04 for $a_{w}$, 0.03-0.04 for moisture content and 22.62-29.51 for total carotenoids content were obtained.

(a)
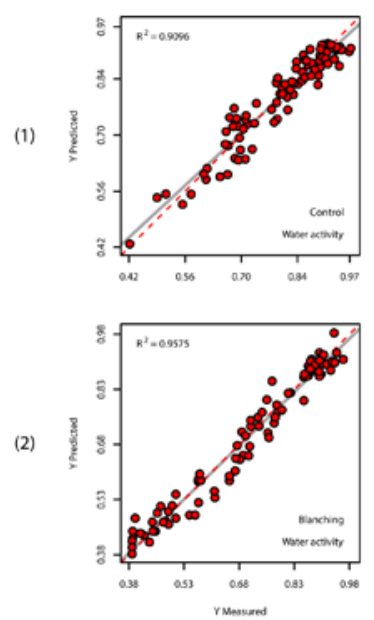

(b)
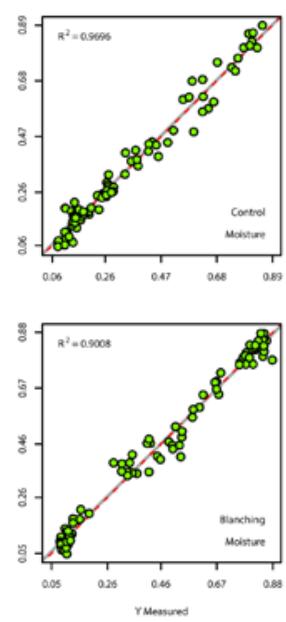

(c)
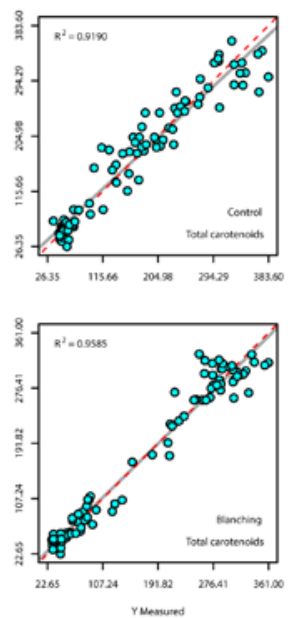

(d)
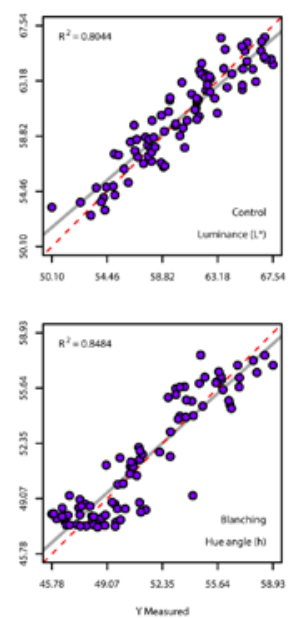

Fig 3: PLS regression plots of Y-measured (reference) and Y-predicted (NIR) values for water activity (column a), moisture (column b), total carotenoids content (column c) and colour changes (column d) monitored during 8-h drying in both unblanched (row 1) and blanched (row 2) carrots. $P L S$ regression plots refer to models based obtained from the full spectrum.

\subsubsection{Regression models (apples)}

PLS regression models with good or excellent predictability were obtained to monitor changes in $a_{w}$ (Fig. 4a), moisture content (Fig. 4b), chroma (Fig. 4c) and SSC (Fig. 4d) of apples wedges during drying, regardless of type of thermal treatment. Except for the calibration model for chroma, the coefficient of determination $\left(R^{2}\right)$ was higher than 0.96 for all the aforementioned quality parameters. Thus, for the test set validation with one quarter of the data (randomly selected) RMSEPs ranging from 0.03-0.04 for $a_{w}, 0.04-0.05$ for moisture content and 4.54-4.99 for SSC were obtained. The latter performance parameter was comparable with both calibration and cross-validation results. 
(a)

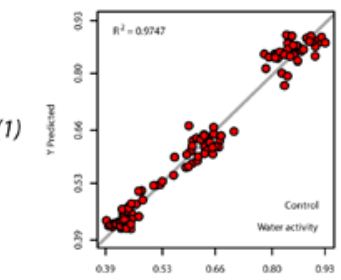

(2)

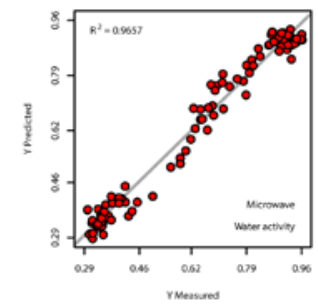

(b)
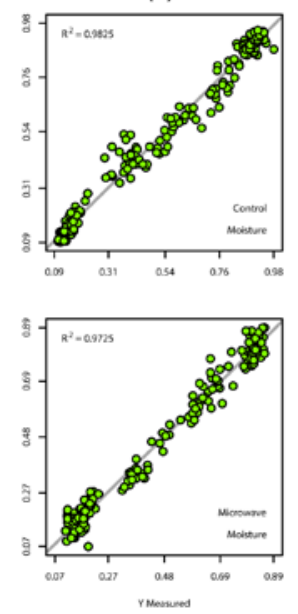

(c)
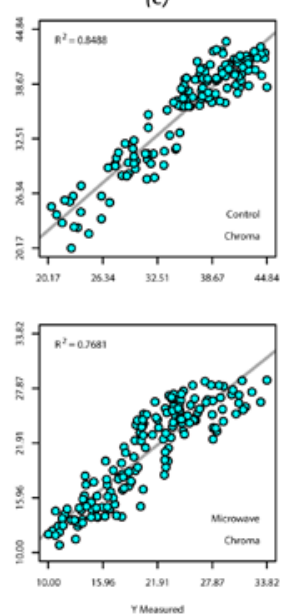

(d)
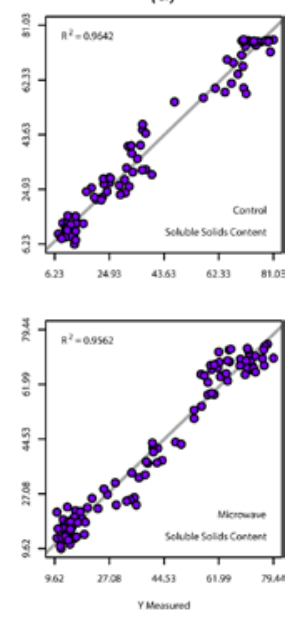

Fig 4: PLS regression plots of Y-measured (reference) and Y-predicted (NIR) values for water activity (column a), moisture (column b), chroma (column c) soluble solids content (column d) monitored during 8-h drying in both control (row 1) and microwave (row 2) treated apples. PLS regression plots refer to models based obtained from the full spectrum.

\section{Conclusions}

In this study, the potential of single-point NIR spectroscopy, Vis/NIR hyperspectral imaging and RGB imaging was evaluated to proactively and non-destructively detect and monitor changes in quality parameters (i.e. water activity, moisture content, total carotenoids content, colours and SSC) of both apples and carrots. For this purpose, regression and classification models were successfully developed obtaining either very good or excellent results in terms of R2 and RMSE. These promising results encourage additional research to develop low-cost dynamic multi factorial process control strategies (based on a Quality by Design approach) using machine learning architectures to produce quality dried products while reducing the environmental impact of the drying processes. Future research should include features not covered by the instrumentation used for this study, increasing the light-beam intensity, and/or combining other chemometric methods, as these could improve the performance of the measurement systems and, thus, the robustness of the smart-drying system under development.

\section{Acknowledgements}

The authors gratefully acknowledge CORE Organic Plus for financial support through the SusOrganic project titled: 'Development of quality standards and optimized processing methods for organic produce’ (Nr: 2814OE006). 


\section{References}

[1] EC. Reg (EC) 889/2008 http://cerescert.com/portal/fileadmin/externdocs/889 2008_compressed.pdf

[2] Wu, D; Sun, D-W. Advanced applications of hyperspectral imaging technology for food quality and safety analysis and assessment: A review - Part I: Fundamentals. Innovative Food Science \& Emerging Technologies. 2013; 19, 1-14.

[3] Sturm, B.; Nunez Vega, A.M.; Hofacker, W. Influence of process control on drying kinetics, colour and shrinkage of air dried apples, Applied Thermal Engineering 2014, 62 (2), 456-460

[4] Sarah, Y. Project Jupyter gets $\$ 6 \mathrm{M}$ to expand collaborative data-science software, http://news.berkeley.edu/2015/07/07/jupyter-project/.

[5] Bantle, M.; Kopp, C., Claussen, I.C.; Tolstorebrov, I. Influence oft he low temperature drying process on optical alternations of organic apple slices. In Proceedings of the $21^{\text {st }}$ International Drying Symposium, Valencia, Spain, September 11-14, 2018

[6] Crichton, S.O.J.; Shrestha, L.; Hurlbert, A.; Sturm, B. Prediction of moisture content and chromaticity of raw and pre-treated apple slices during convection drying using hyperspectral imaging, Drying Technology 2018, doi: 10.1080/07373937.2017.1356847

[7] Savitzky, A.; Golay, M. J. E. Smoothing and Differentiation of Data by Simplified Least Squares Procedures. Analytical Chemistry 1964, 36( 8), 1627-1639.

[8] Boysworth, M. K.; Booksh, K. S. Aspects of multivariate calibration applied to nearinfrared spectroscopy; Burns, D. A.; Ciurczak, E. W. Eds.; Handbook of Near-Infrared Analysis, CRC Press: New York, 2008; 207-229.

[9] de Jong, S. SIMPLS: An alternative approach to partial least squares regression. Chemometrics and Intelligent Laboratory Systems 1993, 18 (3), 251-263.

[10] Moscetti, R.; Saeys, W.; Keresztes, J. C.; Goodarzi, M.; Cecchini, M.; Danilo, M.; Massantini, R. Hazelnut Quality Sorting Using High Dynamic Range Short-Wave Infrared Hyperspectral Imaging. Food and Bioprocess Technology 2015, 8 (7), 1593-1604.

[11] Shrestha, L., Moscetti, R., Crichton, S.O.J., Hensel, O., Sturm, B. Organic apples (cv. Elstar) quality evaluation during hot-air drying using Vis/NIR hyperspectral imaging. In Proceedings of the $21^{\text {st }}$ International Drying Symposium, Valencia, Spain, September 11-14, 2018

[12] Gersdorff, G.; Porley, V.; Retz, S.; Hensel, O.; Crichton, S.O.J.; Sturm, B. (2017). Drying kinetics and quality parameters of dried beef (biltong) subjected to different pre-treatments, Drying Technology, 36 (1), 21-31.

[13] Amjad, W.; Crichton, S.O.J; Munir, A.; Hensel, O.; Sturm, B. Hyperspectral imaging for the determination of potato slice moisture content and chromaticity during the convective hot air drying process, Biosystems Engineering 2018, 166, 170-183.

[14] Moscetti, R.; Sturm, B.; Crichton S.; Massantini, R. Monitoring of organic potato (cv. Anuschka) during hot-air drying using Vis/NIR hyperspectral imaging, Science of Food and Agriculture 2017, doi: 10.1002/jsfa.8737 